\title{
Transient jitter from injection in storage rings
}

\author{
Kai Meng Hock and Andrzej Wolski* \\ Department of Physics, University of Liverpool, Liverpool L69 7ZE, United Kingdom \\ and The Cockcroft Institute, Daresbury, Warrington WA4 4AD, United Kingdom
}

(Received 5 December 2008; published 10 September 2009)

\begin{abstract}
Injection of fresh bunches into a storage ring can induce jitter in the stored bunches. For machines demanding beams of very low emittance and high stability, such as the damping rings of a future linear collider or the storage rings of a super flavor factory, this could be a potential performance limitation. We present an analysis, for the International Linear Collider (ILC) damping rings, of the transverse jitter induced on the extracted bunches from the jitter on the injected bunches, with the coupling between bunches mediated by the resistive-wall wakefield of the vacuum chamber. We find that it is important to include details of the local transverse focusing around the ring. We consider the impact of the finite thickness of the beam pipe wall, and of nonevaporable getter coating on the inside surface: in the parameter regime of the ILC damping rings, we find that the results are only slightly modified compared to an approximation to the resistive-wall wakefield based on a single-layer wall of infinite thickness. The results from our simulations indicate a tight specification on the jitter of the injected bunches.
\end{abstract}

DOI: 10.1103/PhysRevSTAB.12.091001

PACS numbers: $29.20 . \mathrm{db}, 29.27 . \mathrm{Bd}$

\section{INTRODUCTION}

Beam quality and stability are central issues in storage rings and are crucial for the performance of synchrotron light sources, free electron lasers, and accelerators used for high-energy physics. Many applications demand very small beam sizes, with some storage rings recently achieving vertical beams of just a few microns [1-3]. However, as beam sizes become smaller, effects causing variations in bunch trajectories have a greater impact on performance; this can be the case for instabilities caused by ion trapping $[4,5]$, and wakefields [6,7], amongst other effects. While feedback systems have been developed that can suppress a range of instabilities, as beam quality improves and new schemes of operation are developed, residual effects, not completely suppressed by feedback systems, may become important. This may occur, for example, in the damping rings of a future linear collider [8,9], or the storage rings of a future super flavor factory [10]. Both of these machines will demand beams with vertical emittances no more than a few picometers, and bunch-to-bunch position stability at the level of a micron or smaller. Both kinds of machine will also need to maintain the stability of stored bunches while new bunches, which may be subject to considerable variation in bunch-to-bunch position, are injected. In such cases, bunch-to-bunch jitter induced by wakefield coupling between stored and recently injected bunches is a potential concern, because even small effects may adversely impact machine performance, and the time scales involved are beyond the reach of bunch-by-bunch feedback systems.

Transient effects associated with injection, including orbit variation and emittance growth, have been observed in existing storage rings operating with top-up injection

\footnotetext{
*k.m.hock@dl.ac.uk
}

[11]; these effects are generally the result of applied fields from components such as the injection kickers acting on the stored beam. In this paper, we consider the effects arising from wakefield coupling between stored and freshly injected bunches. We apply our analysis and simulations to the damping rings of the International Linear Collider (ILC) [8,9], since the parameter regime of these rings is such that these effects could have a noticeable impact on operational performance. Our goal is to characterize and quantify the bunch-to-bunch transverse jitter on the extracted beam that results from bunch-to-bunch transverse jitter on the injected beam. Since design work on the ILC damping rings is still at a relatively early stage, we have made some assumptions about relevant features, and have only been able to include a single source of longrange (i.e. over the distance of multiple bunches) wakefields; namely, the resistive-wall wakefield arising from the finite conductivity of the beam pipe. Other sources of wakefields, including higher-order modes in the rf cavities, and trapped modes in the vacuum chamber, will need to be included in our calculations when they are known. However, since the resistive wall is expected to make a major contribution to the long-range wakefields in the damping rings, we have attempted to make an accurate estimate by including such features as the finite width of the beam pipe wall, the presence of a coating on the inside surface to improve vacuum and suppress electron cloud, and the variation of the transverse focusing strength on the beam with position around the ring. Also included in the model are details of the operation of the damping rings, taking account of the fill pattern of bunches, and the proposed timing scheme for injection and extraction of bunches.

The novel parameter regime and operating mode of the ILC damping rings means that the effect we are mostly 
concerned with (the jitter on extracted bunches induced by jitter on injected bunches) has not been properly considered previously. Characterizing this effect is the main theme and goal of the work in this paper. Work has previously been published on the resistive-wall wakefield in a vacuum chamber with finite wall thickness [12], and on the effects of variations of focusing strength with position around the ring [13]; and also on techniques for efficient computation of wakefield effects [14]. However, since these issues are highly relevant for our conclusions, we include some discussion as appropriate. In particular, calculation of the wakefield with finite wall thickness and with a coating presents some challenges that require careful consideration. The algorithm we have implemented for computation of the beam dynamics in the presence of the wakefield, based on a Fourier convolution, is not widely known, but is critical for enabling us to perform simulations and obtain results within a reasonable time; and we therefore give some discussion of the implementation of this technique. We have also derived an analytic method for estimating the jitter that is reliable and very fast.

The structure of the paper is as follows. In Sec. II, we describe in more detail the ILC damping rings, giving relevant design features, scheme of operation, and certain aspects of the beam dynamics. In Sec. III, we outline the simulations that we have performed (including discussion of the Fourier convolution method for rapid computation of the wakefield effects) and present our results. In Sec. IV, we discuss the analytic method and results. Finally, in Sec. V we discuss the implications of our results for design and operation of the ILC damping rings.

\section{DYNAMICAL MODEL OF THE ILC DAMPING RINGS}

The ILC damping rings are intended to accept large beams from the electron and positron sources, and reduce their emittances by radiation damping, to produce very low emittance, highly stable beams for acceleration and collision at the interaction point. The beams in the damping rings will be replaced at the machine repetition rate of $5 \mathrm{~Hz}$, with the damping taking place in the $200 \mathrm{~ms}$ between machine pulses. The nominal bunch train in the ILC consists of 2925 bunches, spaced by $332 \mathrm{~ns}$; in the damping rings, the bunch spacing must be reduced to $3.1 \mathrm{~ns}$, so that the train fits into a damping ring of $6.4 \mathrm{~km}$ circumference. During a machine pulse, bunches are extracted individually from each damping ring every $332 \mathrm{~ns}$, to give the correct bunch spacing in the linac. The total extraction process takes around 47 turns. Fresh positron bunches are generated in an undulator-based source $[8,15]$, driven by the electron bunches that proceed to collision. New, undamped positron bunches therefore arrive at the positron damping ring and must be injected, before extraction of the damped bunches is complete. The positron production scheme imposes timing constraints on the overall machine configu- ration and timing scheme, including the fill pattern and the injection/extraction scheme in the damping rings [16]. The nature of the process of production of high-intensity beams of positrons means that substantial jitter is possible. For example, in the baseline positron source [15], high-energy photons travel $500 \mathrm{~m}$ from their production point in a helical undulator to the production target; any residual jitter on the electron beam in the undulator is therefore greatly amplified by the time the photons reach the target. The positron capture and transport sections can also contribute to the jitter, as will the injection system This jitter may be coupled from the injected bunches to stored bunches, through long-range wakefields.

The entire extraction process takes a rather short time, about $1 \mathrm{~ms}$, compared with the $200 \mathrm{~ms}$ of the store time. This raises the possibility of avoiding coupling between damped and freshly injected bunches, by extracting all the damped bunches before injecting any new bunches. However, the fact that positrons are produced by electrons on their way to collision means that, at least for the positron damping ring, there is not the required flexibility for this mode of operation. For the electrons, there will be an independent source, which would allow the possibility of complete extraction before reinjection. But in this case, variations in beam loading while the current in the ring is reduced from $400 \mathrm{~mA}$ to zero are likely to induce more severe transient effects than may arise from coupling between damped and freshly injected bunches. Generally, it is thought desirable to keep variations in beam current in the damping rings as small as possible.

The damping rings will store $400 \mathrm{~mA}$ of beam current at $5 \mathrm{GeV}$ beam energy in a circumference of $6.4 \mathrm{~km}$. With these parameters, coupled-bunch instability is expected, which must be suppressed by a feedback system. Modern coupled-bunch feedback systems provide a damping time of the order of a couple of dozen turns. During a machine pulse, we are concerned with jitter that can build up over 47 turns (driven by the injected beam), so there is a possibility that there will be some residual jitter in the extracted beam, even with a feedback system operating at maximum performance. The vertical beam size in the ILC damping rings will be a few microns, and bunch-to-bunch jitter in the extracted beam must be less than $10 \%$ of the beam size.

The macroparticle model discussed, for example, by Chao [17], has been shown to describe such features as the instability growth rate in storage rings with good accuracy. Assumptions generally made in this model include the following: (i) Each bunch is assumed to behave as a single pointlike charge performing transverse and longitudinal oscillations around the reference trajectory. (ii) The degrees of freedom are uncoupled, so that longitudinal, horizontal, and vertical motion can all be treated independently. (iii) The storage ring is uniformly filled with bunches of equal charge. 
With the above assumptions, simple formulas may be derived to give the growth (or damping) rate of each mode of beam oscillation, in terms of lattice parameters, beam current, and the total impedance of the ring. (A mode in this sense refers to a Fourier mode describing the instantaneous position of each bunch within the ring). Changes in focusing strength around the ring may be accounted to some extent by weighting the impedance at each location around the ring by the local beta function. However, variations in the beta function can lead to additional dynamical effects not taken into account in the usual macroparticle model, and these effects can result in some deviation in the growth rates from the predictions of the simple formulas [13].

The assumption that each bunch behaves as a single pointlike charge is valid for the damping rings, for three reasons. First, the bunch length $(6 \mathrm{~mm})$ is much shorter than the spacing between bunches (of order $1 \mathrm{~m}$ ). Second, the damping rings should operate below threshold for single-bunch instabilities that may increase the transverse or longitudinal dimensions of the bunch [18]. Finally, the betatron phase difference between the head and tail of the bunch, which can result from nonzero chromaticity and would affect the wakefields generated by the bunch, is likely to remain very small. The maximum head-tail phase difference is given approximately by

$$
\Delta \phi \approx 2 \frac{\xi}{\eta} \omega_{0} \sigma_{\tau},
$$

where $\xi$ is the chromaticity, $\eta$ is the phase-slip factor, $\omega_{0}$ the angular revolution frequency, and $\sigma_{\tau}$ the bunch length (in units of time). For the ILC damping rings, the maximum head-tail phase is approximately 0.03 for each unit of chromaticity. Nominally, the chromaticity in the rings should be zero, but there is a possibility that the rings may be operated with one or two units of chromaticity to suppress certain instabilities.

The second assumption, that there is no coupling between the different degrees of freedom, is never exactly satisfied in a real storage ring. There is always some dispersion, which represents coupling between the longitudinal and transverse degrees of freedom, and always some betatron coupling between the transverse degrees of freedom. However, to achieve the specified vertical emittance in the ILC damping rings, both vertical dispersion and betatron coupling must be extremely small; therefore, we assume that we can treat the vertical motion independently of the horizontal and longitudinal. This is fortunate, since the damping rings will be most sensitive to effects in the vertical plane, and it is therefore on this degree of freedom that we focus our attention.

As with many storage rings, the fill pattern in the damping rings will include gaps for ion clearing; the third assumption, therefore, is also not exactly satisfied. Accurate representations of the local focusing and the fill pattern are difficult to include in an analytical model, but are readily accommodated in a simulation. Therefore, in our studies of the wakefield coupling of jitter from injected to extracted bunches, we base our estimates on simulation studies that retain only the first two of the above approximations, i.e. that bunches behave as single pointlike particles, and that vertical motion can be treated independently of horizontal and longitudinal oscillations.

The equation of motion for a bunch in a storage ring relates the acceleration of the bunch to the force from the focusing magnets, and the sum of wake forces from all bunches that have previously passed the present location of the bunch. With the two approximations previously mentioned, plus the approximation that the bunches are moving at the speed of light, the equation of motion for the $m$ th bunch can be written:

$$
\begin{aligned}
M_{1} c^{2} \frac{d^{2} y_{m}}{d s^{2}}= & -M_{1} K(s) y_{m}-q^{2} \sum_{n=1}^{\infty} w_{1}(-c n \tau ; s) \\
& \times y_{m+n}(s-c n \tau),
\end{aligned}
$$

where $y_{m}$ is the displacement of bunch $m$, and $K(s)$ the focusing strength at location $s$ in the accelerator. For convenience, we define $y_{m}$ to be periodic in $m$. So $y_{m+n}$ would be equal to $y_{m^{\prime}}$, where $m^{\prime}$ is the remainder when $m+n$ is divided by $M$, the total number of bunches (including gaps). This simply reflects the fact that the bunches are arranged in a ring. Although there may be gaps in the fill, we assume that all bunches have the same mass $M_{1}$ and charge $q$. In general, the wake force $w_{1}$ depends on the distance between the bunches (assumed separated by integer multiples of the time interval $\tau$ ) and the position in the accelerator. We discuss the wake force in more detail in Sec. III; but here, we note that because the focusing force and the wake force are complicated functions of position, it is in general not possible to integrate analytically of the equations of motion for all the bunches in a storage ring. However, by making the approximation that both the focusing force and the wake function can be replaced by their values averaged around the ring, it is possible to construct equations that can be integrated analytically, and from which useful expressions for tune shifts and instability growth rates can be derived. To avoid this approximation and find more accurate solutions, it is necessary to integrate the equations of motion numerically. Since we are looking for small but potentially significant effects, we follow the latter approach. Before presenting the numerical method, we discuss the wakefield model that we use.

\section{SIMULATION OF COUPLED-BUNCH DYNAMICS}

Calculations of the motion of bunches coupled by wake forces are often based on analytic solutions to the equations of motion [Eq. (1)] obtained using simplifying approximations. Common approximations include: the representation 
of a bunch as a single pointlike charge; the replacement of a structured fill pattern with a uniform distribution of bunches around the ring; and the replacement of the detailed focusing force and the detailed wake force by uniform forces representing respective averages around the ring. Previous work has shown that averaging the focusing force around the ring can lead to the instability growth rates being underestimated. Numerical simulations have the potential to provide greater accuracy, and are also more appropriate for the transient effects with which we are presently concerned.

The simulation technique we shall apply has been described previously [13]. Briefly, we evolve a system of pointlike bunches distributed around the ring in time steps corresponding to the bunch spacing. The lattice functions are used to determine the bunch motion in the absence of any wakefields. At each location in the ring, the wake force is computed from a sum over the history of bunches passing that location, and is used to calculate a kick on each bunch that comes past. The major limitation of this method is the computation time for the wake force sum: the square root dependence on distance means that the wake force dies away slowly, and a history over many turns must be maintained to capture the dynamics accurately. For a large ring with many bunches, such as the ILC damping rings, a direct approach to the computation of the dynamics over 50 turns (corresponding to one extraction and injection cycle) could take many months to complete.

Fortunately, a method using fast-Fourier-transform (FFT) convolution has recently been developed for a similar calculation on the Large Hadron Collider (LHC). In this method [14], the wake forces in the sum are first separated into contributions from individual turns. Each turn in history would contribute a kick. The sum of each turn is then rearranged into the format of a discrete convolution between two arrays of numbers. One array contains the wake function, and the other the bunch displacements. Applying the convolution theorem and the FFT, the computation can be performed much more quickly. We have successfully adapted this method to the ILC damping ring. The computation time is reduced by 2 orders of magnitude, and the simulation can now be done within one day. The details of our adaptation are given in Appendix A.

Apart from some short sections with antechambers in (and some distance downstream of) the dipoles, the vacuum chamber in the ILC damping rings is expected to consist of a simple cylindrical aluminum pipe. The conventional formula for the transverse wake force of such a beam pipe is given, for $z<0$, by [17]

$$
w_{1}(z ; s)=-\frac{2}{\pi b^{3}} \sqrt{\frac{c}{\sigma|z|}}
$$

where $b$ is the radius of the beam pipe and $\sigma$ the electrical conductivity of the wall in the vicinity of the bunch at the time that it experiences the wake force. By causality, the wake force is zero for $z>0$. The formula in Eq. (2) is based on a few assumptions. One is them is that the wall is much thicker than the skin depth. For distances large enough for the skin depth to become comparable to the pipe thickness, the formula in Eq. (2) becomes inaccurate; as will become clear, this is a relevant regime for the effects we are concerned about in the ILC damping rings. We therefore need to consider a more accurate version of this formula, valid over large distances. In recent years, work on the LHC has motivated the development of more accurate calculations of the resistive-wall impedance [12]. The motivation comes from the LHC collimators (which are close to the beam and have poor conductivity), and the multilayered vacuum chambers in many parts of the beam pipe. Using the new methods, we have calculated the impedance for the beam pipe. The wake function is obtained by taking the Fourier transform of the impedance. The result for the finite wall wake function is shown in Fig. 1. Notice the deviation from Eq. (2) for turn numbers that are greater than one.

Using the finite wall wake function results to compute the wake forces, and the FFT convolution technique to sum these wake forces, we can compute the time evolution of the displacement of every bunch. In order to simulate the transient effects associated with injection of bunches with significant transverse jitter, we need to specify the procedure for injection and extraction of bunches. We use a simplified version of the recommendations for the ILC damping ring [16] as follows: (i) Injection and extraction

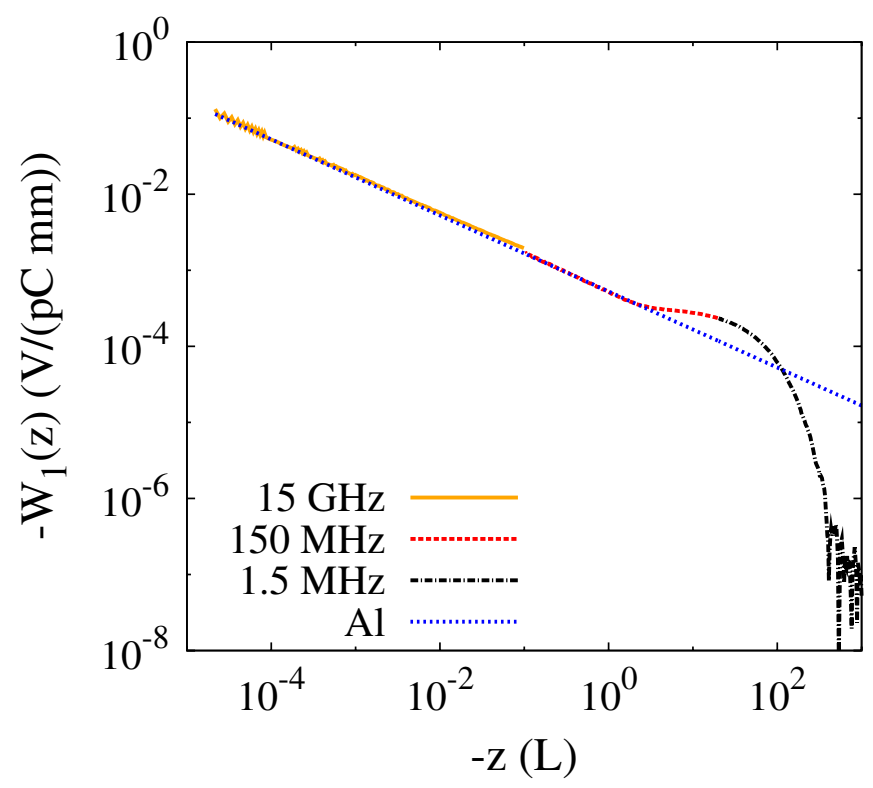

FIG. 1. (Color) The wake function for a beam pipe of radius $3 \mathrm{~cm}$, wall thickness $2 \mathrm{~mm}$, and beam radius $3 \mathrm{~mm}$. The dotted blue straight line is the high-frequency limit obtained from Eq. (2). The curve is finite wall wake function, patched together from the FFT results of three frequency ranges. ( $L$ is the circumference of the ring.) 
TABLE I. ILC damping rings parameters used in simulation studies.

\begin{tabular}{lc}
\hline \hline Harmonic number (circumference) & $14042(6476 \mathrm{~m})$ \\
Beam energy & $5 \mathrm{GeV}$ \\
Beam pipe material (conductivity) & $\mathrm{Al}\left(3.2 \times 10^{17} \mathrm{~s}^{-1}\right)$ \\
Beam pipe radius & $30 \mathrm{~mm}$ \\
Wall thickness & $2 \mathrm{~mm}$ \\
Feedback damping time & $20 \mathrm{turns}$ \\
\hline \hline
\end{tabular}

take place at diametrically opposite sides of the ring. (ii) Bunches are arranged in trains, separated by gaps for ion clearing or electron cloud mitigation. (iii) Extraction starts when bunches are fully damped, i.e. the bunches about to be extracted have zero jitter. (iv) On the first turn, the trailing bunch in each train is extracted in turn. On the second turn, extraction moves on to the next to last bunch on each train. Likewise for the third turn, until all damped bunches are extracted. (v) Injection starts half a turn after the first extraction. Injected bunches fill gaps vacated by extracted bunches, in the same order as the bunches were extracted.

Note that this scheme maximizes the distance between each injected bunch, and the nearest trailing damped bunch. The coupling of jitter from injected to damped bunches through wakefields should therefore be minimized.

In our simulations, we use the current baseline lattice design for the ILC damping rings (DCO2 [9]). The machine parameters are given in Table I, and the specifications for the trains and gaps, also called the fill patterns, are given in Table II. Using these parameters, we simulate the transverse dynamics of bunches under the influence of the resistive-wall wakefield. We are mainly interested in the jitter induced in the damped bunches by the injection of fresh bunches, as this determines the jitter of the extracted bunches. Figure 2 shows the displacement of each bunch immediately before extraction. Notice the increase in jitter with time. Bunches extracted earlier feel the effect of the wake forces from the freshly injected bunches for a shorter time, and are more stable. Bunches extracted at a later time have been under the influence of wake forces from the fresh bunches for a longer time. This greater perturbation results in a larger jitter.

We have assumed an injection jitter corresponding to a coherent betatron amplitude that is $10 \%$ of the specified maximum betatron amplitude $(0.09 \mathrm{~m} \mathrm{rad})$ of the particles

TABLE II. Fill patterns used in simulation studies.

\begin{tabular}{lcc}
\hline \hline & Pattern I & Pattern II \\
\hline Bunches per train & 45 & 45 \\
Bunch spacing (rf buckets) & 2 & 2 \\
Bunch population $\left(\times 10^{10}\right)$ & 1.0 & 1.9 \\
Intertrain gap (rf buckets) & 30 & 126 \\
\hline \hline
\end{tabular}

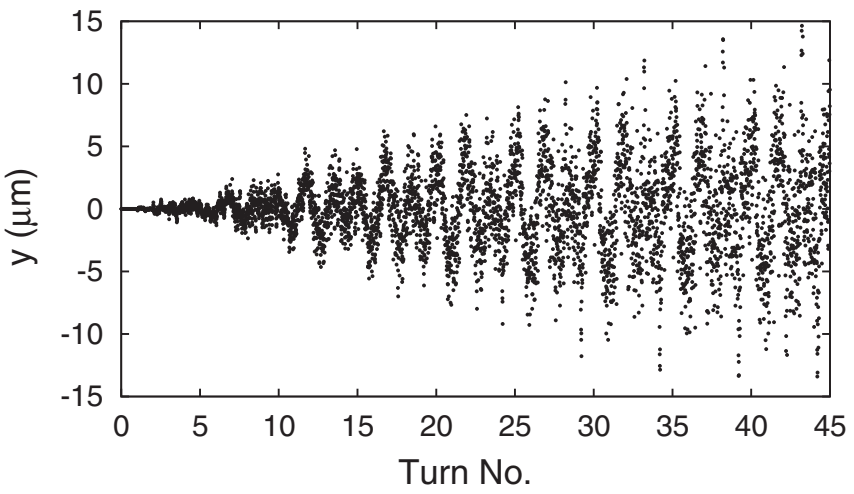

FIG. 2. The vertical jitter (displacement) of each bunch at the extraction location is recorded, just before it is extracted. The horizontal axis is the time, in units of revolution time.

in the injected beam. For example, if the bunches are injected at a location on the ring with a vertical beta function of $16.79 \mathrm{~m}$, the vertical injection offsets would have a Gaussian distribution with an rms of about $1 \mathrm{~mm}$. The extraction jitter in Fig. 2 is obtained from one particular set of random vertical offsets for the injected bunches.

For a more complete physical picture, note that the injection and extraction take place over a period of 45 turns. Consider an ideal case when the ring is completely filled with 7021 bunches, and when the beta function has a constant, averaged value of $16.79 \mathrm{~m}$. The resistive-wall instability would give a maximum growth mode with a growth time of about 75 turns. In contrast, the multibunch feedback system has a damping time of 20 turns. This is enough to control the instability in the ideal case with no injection and extraction. However, as Fig. 2 shows, the feedback system is clearly not strong enough to suppress the jitter induced by the wakefields of the injected bunches. The radiation damping time in this machine is $25 \mathrm{~ms}$, which is just over 1000 turns. This is much longer than the time scale of injection and extraction, so the effect may be neglected.

We also investigated the effects of changes in the injection-extraction procedure; for example, we find that if extraction and injection start with the leading bunch of each train, instead of with the trailing bunch, the jitter is increased by about $20 \%$.

To preserve luminosity in the ILC, the specification on the damping rings extraction jitter is that the rms centroid displacement should be less than $10 \%$ of the rms beam size. In Fig. 2, the rms centroid displacement is $50 \%$ of the rms beam size. Since the equations of motion for the bunch coordinates are linear, to stay within the specified limit on the extraction jitter, our results show that the injection jitter should be less than $0.2 \mathrm{~mm} \mathrm{rms,} \mathrm{or} 3 \%$ of the specified beam envelope.

This specification may be difficult to achieve, given the nature of positron production. However, it may be possible to relax this specification, by installing a feed-forward 


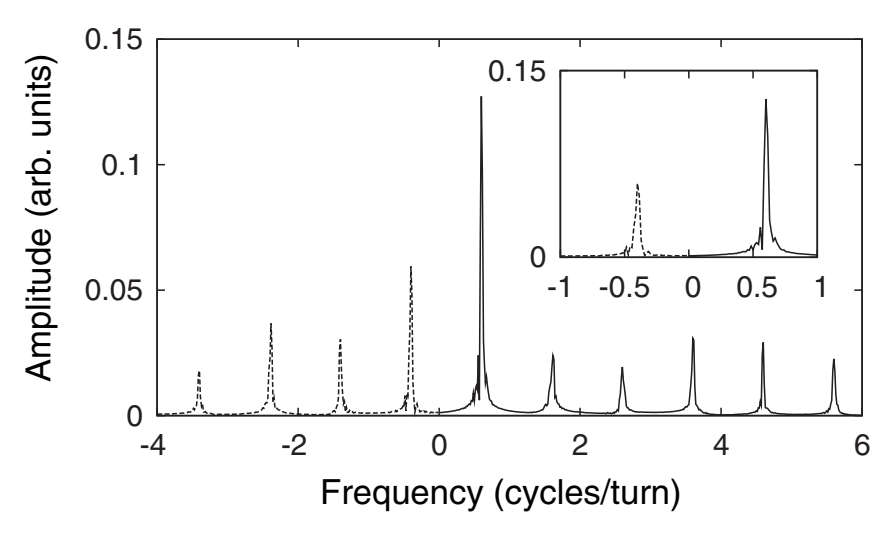

FIG. 3. Spectrum of the extraction jitter. The horizontal axis is the frequency in cycles per turn. The inset shows the highest peak with a magnified horizontal scale.

system to suppress the jitter after the bunches have been extracted. Such a system would apply kicks to the beam, determined by signals from beam position monitors. Inspecting the jitter results in Fig. 2, we observe certain characteristics that could be exploited for this purpose. Although we specified random values for the injection offsets, there is a clear modulation in the extraction jitter. This modulation is very slow, with a dominant frequency less than one cycle per turn. The spectrum of the extraction jitter is shown in Fig. 3, and displays a clear peak at 0.6 cycle/turn that indicates a dominant, sinusoidal oscillation mode. If this is a universal feature, it would suggest a feed-forward system that uses just one frequency to suppress the strongest modes. This would be simpler and cheaper to build than a bunch-by-bunch system, which requires a wide bandwidth and complex electronics. Unfortunately, there is a significant contribution from other modes. A simple subtraction of the dominant mode in Fig. 2 shows that much of the jitter remains. The "background" peaks in Fig. 3 are not small. In Appendix B, we propose an explanation for this modulation, which suggests that it is indeed characteristic of the extraction jitter for all machines with wakefield coupling between bunches.

Finally, we compare our results with those that would have been obtained using the usual macroparticle model. Figure 4 shows the results for the three cases in Table III. Case I is the usual macroparticle model, with uniform focusing and the thick-wall approximation for the wake function. As mentioned above, variation in the focusing around the ring may be taken into account to some extent by weighting the wake function with the local beta function. In the present case, since the beam pipe aperture and conductivity are uniform around the ring, performing the average of the wake function weighted by the beta function simply gives the wake function multiplied by the average beta function: it is this average beta function that is used in the model in case I. The amplitude of the jitter found using this model is clearly smaller than the jitter in the other two cases. This shows that variation of the focusing, as occurs
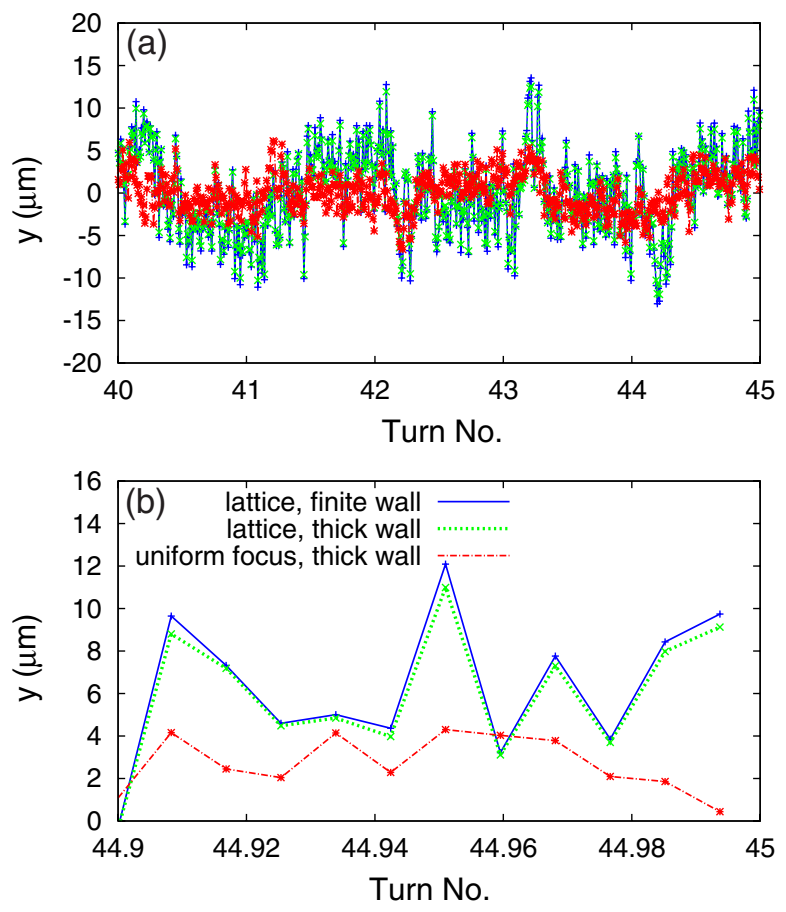

FIG. 4. (Color) (a) The dashed red line shows the jitter computed with the approximation of uniform focusing, and using the wake force in the high-frequency limit (case I). The dotted green line again approximates the wake function using the highfrequency limit, but uses the actual lattice functions (case II). The solid blue line shows the jitter for the actual lattice and a beam pipe with finite wall thickness (case III). (b) The horizontal scale is magnified to show the relative magnitude of the jitter in each case.

in a real lattice, has an impact on the dynamics that is not negligible. The amplitude of the jitter given by the finite wall wake function (case III) is slightly higher than that for the high-frequency limit (case II). Although the finite wall wake function is larger than the high-frequency limit between 1 and 100 turns (as shown in Fig. 1), below 1 turn the wake functions are very close. This suggests that the dominant contribution to the sum of wake forces comes from leading bunches that are within a distance of a single turn in the ring. For a wall thickness of $0.2 \mathrm{~mm}$, the peak in the finite-wall wake function would move below the length scale of a single turn; at that point, we would expect to see some significant effect on the jitter from the finite thickness of the beam pipe wall. However, it is not expected that the wall thickness in the ILC damping rings will be less than $2 \mathrm{~mm}$.

TABLE III. Conditions for wake function and jitter calculations.

\begin{tabular}{lcc}
\hline \hline Case & Focusing strength & Wake function \\
\hline I & Uniform & High-frequency limit \\
II & Actual lattice & High-frequency limit \\
III & Actual lattice & Finite wall \\
\hline \hline
\end{tabular}




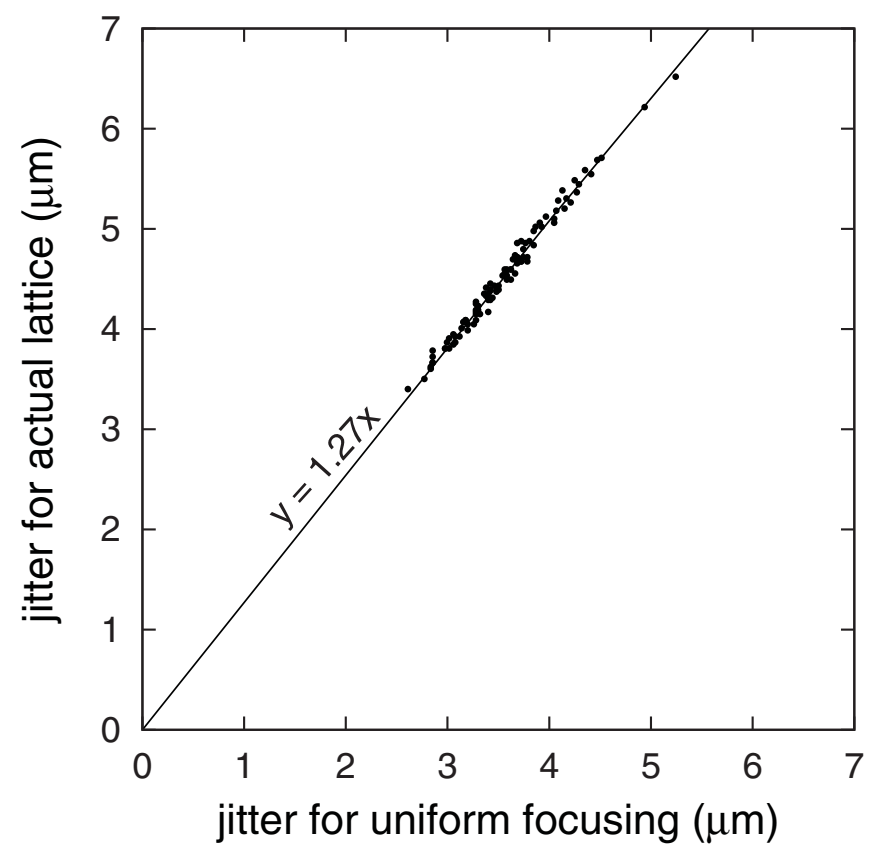

FIG. 5. Correlation between extraction jitter assuming uniform focusing, and for the actual lattice with local focusing, for 100 seeds of random injection jitter.

For a more complete picture of the effect of the actual lattice on the jitter, we repeat the simulation for 100 different sets of injection jitter. For each set, we do the simulation for both the actual lattice and the averaged lattice with uniform focusing. The resulting rms jitters are shown in Fig. 5. The excellent correlation shows that the standard model with the assumption of uniform focusing is a reasonable approximation. However, there is indeed a significant increase in jitter as a result of the local focusing in the actual lattice, in this case by $27 \%$.

\section{ANALYTIC ESTIMATION OF TRANSIENT JITTER}

In the system described so far, potentially thousands of bunches are involved. The complexity of the problem suggests that a full simulation is required, which is computationally intensive. It is therefore surprising to find that an analytic solution, which can be rapidly computed, is in fact possible.

We are concerned with coherent transverse motion of bunches of electrons or positrons. In this case, it is possible to represent each bunch as a single pointlike particle with charge equal to the total charge of the bunch; this model has been widely used and shown to be reliable $[17,19,20]$. Decoherence may be accounted as an additional damping effect; the emittance dilution arising from decoherence is not considered here. We assume that all bunches in the ring have been damped into a stable, narrow beam before injection of fresh bunches begins. Injection errors can result in the injected bunches having random transverse offsets from the design path; these offsets cause wakefields that perturb the trailing bunches, resulting in random bunch-to-bunch offsets, or "jitter" of all bunches in the ring. To determine the transverse displacement $y_{m}$ of the $m$ th bunch just before it is extracted, we write down the equation of motion in a more standard form as follows $[17,21]$ :

$$
\begin{aligned}
\frac{d^{2} y_{m}}{d t^{2}}+2 \zeta \frac{d y_{m}}{d t}+\omega_{\beta}^{2} y_{m}= & -\frac{N r_{0} c}{\gamma T_{0}} \sum_{n=1}^{\infty} W_{1}(-c n \tau) \\
& \times y_{m+n}(t-n \tau),
\end{aligned}
$$

where $\omega_{\beta}$ is the betatron frequency, $N$ the average bunch population, $r_{0}$ the classical electron radius, $\gamma$ the electron energy in units of its rest energy, $T_{0}$ the revolution time, $\tau$ the time for a bunch to traverse the bunch spacing, and $W_{1}(z)$ the transverse wake function. $\zeta$ is a damping constant representing the combined damping effects of synchrotron radiation, the transverse feedback system, and decoherence. Note that we also use here the definition that $y_{m}$ is periodic in $m$.

In order to solve Eq. (3) analytically in the presence of injection and extraction, note that the injection offsets are much larger than the displacements of the damped bunches. The typical jitter after damping is less than $1 \%$ of the injection offsets, as the simulation results show later. The effect of damped bunches on injected bunches is small because their displacements are small. The coupling of injected bunches on damped bunches may therefore be treated as a driving force. We also assume that the effect of coupling among injected bunches is substantially reduced because they are not all injected right from the start. This suggests that the dominant coupling is between injected and damped bunches. In this model, we shall only consider this effect. With this simplification, the equation of motion may be written in the following form:

$$
\frac{d^{2} y_{m}}{d t^{2}}+2 \zeta \frac{d y_{m}}{d t}+\omega_{\beta}^{2} y_{m}=A f_{m}(t) e^{-i \omega t},
$$

where $A$ and $f(t)$ are explained as follows.

The right-hand side of Eq. (4) is the sum of the wake forces from only the injected bunches on the damped bunch $m$. To obtain this sum of wake forces from the right-hand side of Eq. (3), consider an injected (i.e. undamped) bunch $n$. This bunch must be subject to the same feedback damping and focusing forces as the damped bunches, except that there would be no driving force. Thus it must obey Eq. (4) with the right-hand side set to zero. The displacement of injected bunch $n$ may therefore be written as

$$
y_{n}\left(t^{\prime}\right)=a_{0} z_{n} e^{-\zeta t^{\prime}} e^{-i \omega t^{\prime}},
$$

where $\omega=\sqrt{\omega_{\beta}^{2}-\zeta^{2}} \cdot a_{0}$ is the rms of the injection offset, and $z_{n}$ is a complex number whose real and imaginary parts are random numbers with a Gaussian distribution of rms 1 . 
The exponential factors take into account, respectively, the damping processes and the betatron oscillation. The time, $t^{\prime}$, starts from the time of injection.

Substituting this into Eq. (3), the right-hand side can be rearranged into the form in Eq. (4). The constant $A$ contains physical constants such as the charge and mass of the particles, and fixed parameters associated with the machine design, such as the particle energy and the revolution period. The function $f(t)$ contains the time dependence of the wakefields and the bunches generating the wakefields.

The wakefields come from the resistive-wall effect; the fields at a distance $z$ behind a pointlike charge may be derived from the wake function, $W_{1}(z)$. This may be computed using a thick wall approximation [17], or more sophisticated methods [12]. Define

$$
A=\frac{N r_{0} c}{\gamma T_{0}} a_{0}
$$

and

$$
w(n)=-W_{1}(-n d),
$$

where $d$ is the bunch spacing.

To derive an expression for $f(t)$, suppose that we start with perfectly damped bunches. The nature of the wake function $W_{1}$ is such that a damped bunch that just passes the injection location at $t=0$ will experience only wake forces from bunches injected ahead of it:

$$
f_{m}(0)=\sum_{n>m} q_{n} z_{n} \delta_{1 k_{n}} w(n-m),
$$

where $n>m$ if bunch $n$ is ahead of bunch $m$; bunch $n$ is injected on turn $k_{n}$; and $q_{n} N$ is the actual population of bunch $n$. The Kronecker delta function $\delta_{i j}$ is 1 if $i=j$, and 0 otherwise. In Eq. (8) and in the next three equations, $n$ refers only to injected bunches. A summation over the index $n$, such as the one in Eq. (8), is to be understood as a summation over injected bunches only. The Kronecker delta function is used to determine the particular turn in which the bunches are injected. For instance, the presence of $\delta_{1 k_{n}}$ means that the sum only needs to be taken over bunches that are injected during the turn number $k_{n}=1$. Note also that the turn number 1 starts at a time when the bunch number 0 passes the injection point, and ends when the bunch number 0 passes the injection point again. This means that turn 1 starts at $t=-(M-m) \tau$, and ends at $t=m \tau$.
At the start of the second turn, bunch $m$ starts to see the wakefields generated by all undamped bunches over the first turn, plus the wakefields generated by undamped bunches ahead of it in the second turn. The total wakefield can be written as

$$
\begin{aligned}
f_{m}\left(T_{0}\right) e^{-i \omega T_{0}}= & \sum_{n} q_{n} z_{n} \delta_{1 k_{n}} w(n-m+M) \\
& +\sum_{n>m} q_{n} z_{n} \delta_{1, k_{n}} w(n-m) e^{-i \omega T_{0}} e^{-\zeta T_{0}} \\
& +\sum_{n>m} q_{n} z_{n} \delta_{2, k_{n}} w(n-m) .
\end{aligned}
$$

At this time, some of the bunches injected during the first turn (those for which $n>m$ ) have gone around the ring more than once. These bunches contribute twice to the wakefield at the injection point. The first contribution comes from these bunches passing by the injection point the first time. The second contribution comes from these same bunches passing by the injection point the second time. In Eq. (9), the first term on the right comes directly from all bunches injected during turn 1, and includes the first contribution. The next term contains only the second contribution. When these bunches ( $n>m$ from turn 1) pass the injection point the second time, their displacements are quite different from the first time. They have undergone betatron oscillation and feedback damping for time $T_{0}$, which explains the appearance of the two exponential factors. The third term comes from bunches injected from the start of turn 2 up until the current time $t=T_{0}$. Continuing the reasoning leads to the formula:

$$
\begin{aligned}
f_{m}\left(n_{t} T_{0}\right)= & \sum_{i_{t}=1}^{n_{t}} \sum_{j_{t}=i_{t}}^{n_{t}} \sum_{n} q_{n} z_{n} \delta_{i_{t} k_{n}} \\
& \times w\left[n-m+\left(n_{t}-j_{t}+1\right) M\right] \\
& \times e^{-i\left(j_{t}-i_{t}-n_{t}\right) \omega T_{0}} e^{-\left(j_{t}-i_{t}\right) \zeta T_{0}} \\
& +\sum_{i_{t}=1}^{n_{t}+1} \sum_{n>m} q_{n} z_{n} \delta_{i_{t} k_{n}} w(n-m) e^{-i\left(1-i_{t}\right) \omega T_{0}} \\
& \times e^{-\left(n_{t}+1-i_{t}\right) \zeta T_{0}} .
\end{aligned}
$$

The wakefields from all injected bunches are included in the sum over $i_{t}$; the wakefields from all turns are included in the sum over $j_{t}$. By regrouping terms, we find that Eq. (10) can be written as an iterative formula, for $n_{t}>0$ :

$f_{m}\left(n_{t} T_{0}\right)=f_{m}\left[\left(n_{t}-1\right) T_{0}\right] e^{-\zeta T_{0}}+e^{i \omega n_{t} T_{0}}\left(\sum_{i_{t}=1}^{n_{t}} \sum_{n} q_{n} z_{n} \delta_{i_{t} k_{n}} w\left[n-m+\left(n_{t}+1-i_{t}\right) M\right]+\sum_{n>m} q_{n} z_{n} \delta_{n_{t}+1, k_{n}} w(n-m)\right)$

The equivalence of Eqs. (10) and (11) can also be shown by repeated substitution in Eq. (11), starting with $f_{m}(0)$ given by Eq. (8). The iterative expression for $f_{m}\left(n_{t} T_{0}\right)$ given by Eq. (11) is much faster to compute than the alternative expression, Eq. (10). Finally, we note that, taking into account betatron oscillations and damping, the driving term $f_{m}(t)$ at an arbitrary 
time $t=n_{t} T_{0}+\Delta t$ can be found from

$$
f_{m}(t)=f_{m}\left(n_{t} T_{0}\right) e^{-\zeta \Delta t},
$$

where $n_{t}$ is an integer, and $\Delta t<T_{0}$.

Equation (4) can now be solved analytically over the duration of each turn. The solution is given by

$$
y_{m}(t)=A_{n_{t}} e^{(-\zeta+i \omega) t}+B_{n_{t}} e^{(-\zeta-i \omega) t}+p_{n_{t}} t e^{(-\zeta-i \omega) t},
$$

where

$$
\begin{gathered}
p_{n_{t}}=-A f_{m}\left(n_{t} T_{0}\right) \frac{e^{n_{t} \zeta T_{0}}}{2 i \omega}, \\
\omega=\sqrt{\omega_{\beta}^{2}-\zeta^{2}} .
\end{gathered}
$$

$A_{n_{t}}$ and $B_{n_{t}}$ are constants that can be determined by matching $y_{m}$ and $d y_{m} / d t$ between adjacent turns: at $t=n_{t} T_{0}$, where solutions from two sides meet, $y_{m}$ and $d y_{m} / d t$ must be continuous. Imposing this condition, assuming that the damped bunches have zero transverse displacements and momenta initially, and using a set of random injection offsets, the full solution can be computed.

The simulation technique has been described in the previous section. Using the same parameters, we simulate the transverse dynamics of bunches in the presence of the resistive-wall wakefield. We are mainly interested in the jitter induced in the damped bunches by the injection of fresh bunches, as this determines the jitter of the extracted bunches. The amplitude of each bunch immediately before extraction is determined from the detailed simulation. The jitter is then defined as the root mean square of the amplitudes of all the extracted bunches. Figure 6 shows the jitter calculated analytically using Eq. (13), plotted against the jitter obtained by simulation, for 100 different sets of random injection offsets. The analytic results are higher than the simulated results by $11 \%$. This may be attributed to the coupling that has been neglected. The good correlation in Fig. 6 demonstrates the reliability of the analytic formula. We have used the thick wall approximation for the wake function [17], which is valid in many regimes of interest. Using Eq. (13), the computation time is reduced, compared with simulation, from one day to 1.5 minutes on our Pentium $3.40 \mathrm{GHz}$ computer.

Next, consider the range of validity of the analytic method with respect to the strength of the wakefield, determined by the factor $A$ in Eq. (4). In the analytic method, the jitter increases linearly with $A$. We carried out a number of simulations for increasing bunch population, $N$, which is related to $A$ by Eq. (6). The simulated jitter starts to deviate from the analytic jitter when the rms value reaches $100 \mu \mathrm{m}$. This is about $10 \%$ of the rms of the injection offsets that we used. If the jitter of the damped bunches is as big as the injection offsets, then the coupling among the damped bunches would become comparable to

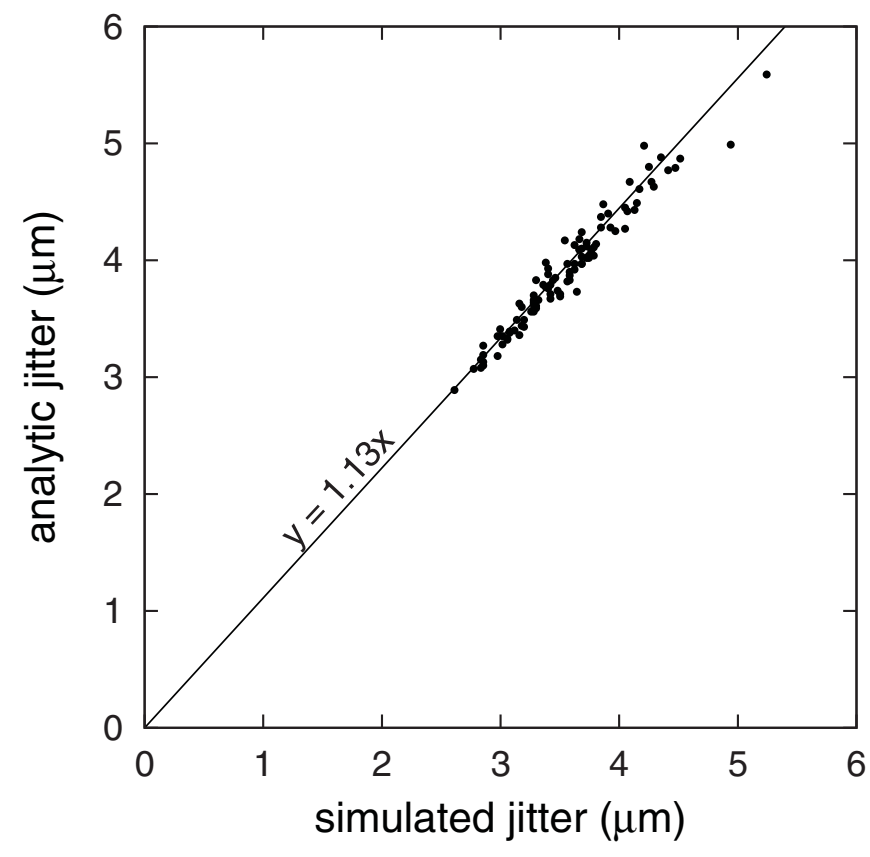

FIG. 6. Correlation between extraction jitter calculated analytically and found from simulation, for 100 seeds of random injection jitter.

the coupling with the injected bunches, and the assumptions would no longer be valid. Thus, it is reasonable to expect that the analytic results start to deviate when the jitter exceeds $10 \%$ of the injection offsets. For the current machine parameters, this takes place when $N$ reaches $2 \times$ $10^{11}$, which corresponds to 8 amperes.

Although both problems - the simulation and the analytic estimation-are linear, this deviation occurs because of a change in the nature of the solutions. The solutions to Eq. (3) are exponential functions-they are exponential in $N$, which appears as a factor in the growth rate [17]. The solution to Eq. (4), on the other hand, is linear in $N$. When the jitter in the damped bunches reaches $100 \mu \mathrm{m}$, it starts to be comparable to the offsets in the injected bunches. The assumption of negligible coupling from the damped bunches begins to break down. This means that we need to switch back from Eq. (4) to Eq. (3). So the solution switches from linear to exponential. Hence the deviation.

In our simulations, we assumed an injection jitter corresponding to a coherent betatron amplitude that is $10 \%$ of the specified maximum betatron amplitude $(0.09 \mathrm{~m} \mathrm{rad})$ of the particles in the injected beam. Figure 7 shows a histogram of the analytic jitter, for 1000 different sets of random injection offsets. This calculation takes one day on our computer, whereas a full simulation would take nearly three years. Such statistics are important-the spread in Fig. 7 means that, if only one sample is taken, it is easy to overestimate or underestimate the jitter by a few times. After correcting for the $11 \%$ difference in Fig. 6, we obtain a mean jitter of $63 \%$, and a standard deviation of $18 \%$. Both percentages are given relative to the specified vertical 


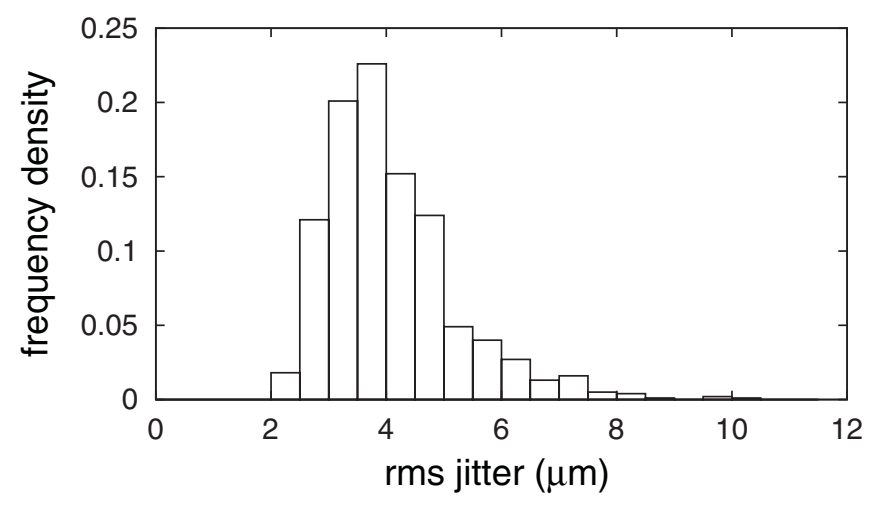

FIG. 7. Distribution of rms extraction jitter, for 1000 seeds of random injection jitter.

emittance of $2 \mathrm{pm}$. The mean jitter is more than half the bunch size: if transported to the interaction point, this jitter would mean that many bunches would fail to collide. To maintain luminosity, the bunch-to-bunch jitter needs to be no more than $1 \%$ or $2 \%$ of the beam size. This criterion can be used, in conjunction with the analytical methods presented in this paper, to specify the maximum allowable jitter on the injected bunches.

\section{CONCLUSIONS}

Jitter on the beam extracted from the damping rings could have an impact on the luminosity in the ILC. We have developed a method for modeling the jitter induced on the extracted bunches from coupling through wakefields to the jitter on injected bunches. We find that the approximation of uniform focusing underestimates the extracted jitter. However, using a form for the resistive-wall wake function based on a beam pipe wall of infinite thickness appears to be a good approximation in the parameter regime of the damping rings; only if the wall thickness is reduced to the order of $0.2 \mathrm{~mm}$ (substantially below the present specification of $2 \mathrm{~mm}$ ) will the finite thickness of the beam pipe wall have an impact on the beam dynamics. Similarly, the nonevaporable getter coating will likely not affect the coupled-bunch motion.

The results of our simulations allow us to specify a limit on the injection jitter, given that the injection jitter should be less than $10 \%$ of the beam size. For a beta function of $5 \mathrm{~m}$ at the injection point, the vertical jitter on the injected bunches should be less than $0.2 \mathrm{~mm}$. Although this may be difficult to achieve for the positron beam, it is possible that a feed-forward system in the extraction line may be used to relax the specification on the injection jitter, by correcting the extraction jitter. Such a system could be based on the fact that the jitter on the extracted bunches is dominated by a few modes with large growth rates in the damping rings, and therefore has a large systematic component.

\section{ACKNOWLEDGMENTS}

We are grateful to the Science and Technology Facilities Council for funding. We would like to thank Daniel Schulte and Alexander Koschik for drawing our attention to the use of the FFT convolution method for rapid calculation of the wakefield sum, and for helpful discussions regarding the application of this technique.

\section{APPENDIX A: SUMMATION OF WAKE FORCES}

Summing of the wake forces for every bunch and at every time step is the most computationally intensive part of the simulation reported in Sec. III. We have adapted the method of Koschik [14] in order to speed up the computation. The sum of wake forces must first be rearranged into the form of a discrete convolution [22]:

$$
(r * s)_{j}=\sum_{k=N / 2+1}^{M / 2} s_{j-k} r_{k},
$$

where the array $s$ may be described as the signal, array $r$ the response, and $N$ is the size of the response array. This convolution can then be computed by applying an FFT to each array, multiplying the results, then inverting the FFT. In order to see that this is possible, consider the sum for each bunch explicitly, as shown in Table IV.

Compare this with the expression above for discrete convolution. Notice that the wake function "moves" through the bunch displacements as we go from bunch 0 to bunch 1, and from bunch 1 to bunch 2. This is similar to the way that the response moves through the signal in Eq. (A1). So the sum of wake forces is indeed a convolution.

However, it is not necessary to apply FFT to the full history which, in our case, consists of displacements for the 50 turns required for convergence. The reason is that at every time step, we only require the sum of wake forces for the $M$ bunches at that time step. Given a minimum bunch separation of two rf buckets in the ILC damping rings, the number of bunches is potentially as large as half the harmonic number of the rings, which is 14042 for the machine design used in this paper. Thus, $M$ can be up to

TABLE IV. Some of wake forces on the first three bunches.

\begin{tabular}{lc}
\hline \hline Bunch & Sum \\
\hline 0 & $W_{1}(-c \tau) y_{1}(t-\tau)+W_{1}(-2 c \tau) y_{2}(t-2 \tau)+W_{1}(-3 c \tau) y_{3}(t-3 \tau)+W_{1}(-4 c \tau) y_{4}(t-4 \tau)+\cdots$ \\
1 & $W_{1}(-c \tau) y_{2}(t-2 \tau)+W_{1}(-2 c \tau) y_{3}(t-3 \tau)+W_{1}(-3 c \tau) y_{4}(t-4 \tau)+\cdots$ \\
2 & $W_{1}(-c \tau) y_{3}(t-3 \tau)+W_{1}(-2 c \tau) y_{4}(t-4 \tau)+\cdots$ \\
\hline \hline
\end{tabular}


7021. We can of course perform the convolution for the full history array with a size of $50 \times 7021$, but we would then only use the first 7021 values in the final result and discard the rest. Computationally, it is far more economical to divide the convolution sum into separate sums of $M$ terms, each corresponding to a turn in history, perform the Fourier convolution for each turn, then summing up the results from every turn.

Note that in Table IV, the bunch displacements do not refer to the same point in time. Each bunch is displaced by one step in time with respect to the next one. This is how the history has to be stored, in order to apply the Fourier convolution. It is a different format from the straightforward integration of the equations of motion that is used in [13], in which bunch displacements at the same point in time are stored at each integration step. This new format is in fact not a disadvantage. The equations can still be integrated in the same way at every integration step. As bunch 1 is one time step earlier than bunch 0 , there is the concern the history of bunch 1 that is required for the wake force on bunch 0 is not yet available. In fact, because both the wakefield and bunches travel at or close to the speed of light, the wake force from the displacement of bunch 1 that is recorded at the same integration step would arrive bunch 0 just in time. Likewise for the other bunches. In Ref. [13], the Fourier modes were computed from the normalized action of every bunch at the same instant in time. With the new format, the same Fourier modes can be computed by multiplying the normalized action of each bunch by a corresponding phase factor in time.

This approach provides a particular advantage in the simulation of injection and extraction. Although the displacements in Table IV do not refer to the same time step, they do refer to the same location on the ring-if we consider $M$ locations separated uniformly by the bunch spacing. This means that at each integration step, we are in effect moving by one step along the ring. The new displacements obtained from the integration are effectively the displacements of the bunches as they pass through a given location in one turn. This means that we can obtain the displacement of each bunch at the extraction location at the same step of the integration. Likewise, when we reach the injection location, all bunches can be "injected" at the same integration step. This simplifies the coding process considerably.

To implement the Fourier convolution separately on each turn of history, it is necessary to pad zeros at the right places in the arrays. Consider the $n$th turn of history. The displacements are given by $y_{0}\left(t-n T_{0}\right), y_{1}(t-\tau-$ $\left.n T_{0}\right), \ldots, y_{M-1}\left(t-\tau M-1-c n T_{0}\right) . T_{0}$ is the time for one revolution around the ring. This same set of values has to be multiplied by the wake function for each bunch, as shown in Table V.

To improve readability, we have defined $\tau_{k}=k \tau$ and $z_{k}=k c \tau$ for time steps and location steps. In order to apply a Fourier convolution to this, the following arrays may be zero padded and formatted as follows:

$$
\begin{aligned}
& s_{j}= \begin{cases}y_{j}\left(t-\tau_{j}-n T_{0}\right), & \text { for } j=0, \ldots, M-1, \\
0, & \text { for } j=-(M-1), \ldots,-1 .\end{cases} \\
& r_{k}=W_{1}\left(z_{k}-n L\right), \quad \text { for } k=-(M-1), \ldots, M-1 .
\end{aligned}
$$

In the resulting convolution, $(r * s)_{j}$, the first $M$ values of $j$ from 0 to $M-1$ would give the wake forces for the corresponding bunches. Note that the arrays for the first turn, or the 0th turn as we have defined it, may look slightly different. The reason is that half of the array $r$ would be zero because the arguments of the wake function are positive. Otherwise, the same formulas for the arrays can be used. Using these arrays, the contributions to the wake forces from each turn in history can be evaluated. These are then summed for all turns in history to give the total wake force on every bunch.

\section{APPENDIX B: DOMINANT JITTER MODES}

In Sec. III, a clear modulation was observed in the extraction jitter. In order to explain this, recall that for the case of uniform focusing-or constant beta function as described in [13] — the bunch displacements can be resolved into Fourier modes that either grow or decay exponentially. Figure 8 shows the growth rates of some of the Fourier modes in the DCO2 damping ring. The random displacements of a set of bunches can be expressed as a linear combination of Fourier modes. Over time, some of these will decay, while others will grow. After a sufficient period, only the modes with the highest growth rates will be visible. Thus, starting from a random distribution displacements, we would end up with a very clear correlation among bunches, with a profile resembling a sine wave.

TABLE V. Convolution of displacements and wake functions in the $n$th turn of history.

\begin{tabular}{lc}
\hline \hline Bunch & Sum \\
\hline 0 & $W_{1}\left(z_{0}-n L\right) y_{0}\left(t-\tau_{0}-n T_{0}\right)+\cdots+W_{1}\left(z_{-(M-1)}-n L\right) y_{M-1}\left(t-\tau_{M-1}-n T_{0}\right)$ \\
1 & $W_{1}\left(z_{1}-n L\right) y_{0}\left(t-\tau_{0}-n T_{0}\right)+\cdots+W_{1}\left(z_{-(M-2)}-n L\right) y_{M-1}\left(t-\tau_{M-1}-n T_{0}\right)$ \\
$\cdots$ & $\cdots$ \\
$M$ & $W_{1}\left(z_{M-1}-n L\right) y_{0}\left(t-\tau_{0}-n T_{0}\right)+\cdots+W_{1}\left(z_{0}-n L\right) y_{M-1}\left(t-\tau_{M-1}-n T_{0}\right)$ \\
\hline \hline
\end{tabular}




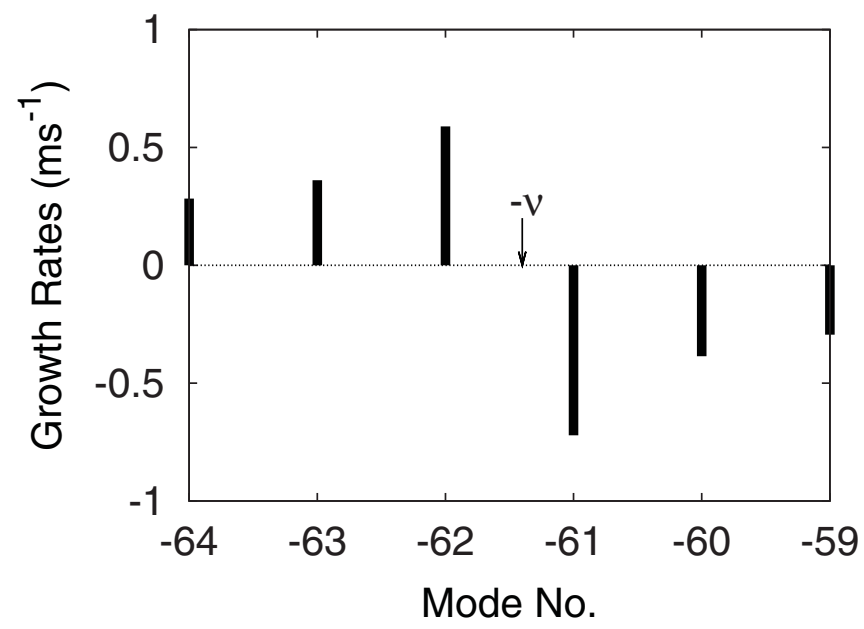

FIG. 8. The growth rates of some of the transverse, multibunch Fourier modes in the DCO2 damping ring, assuming uniform focusing, calculated using the analytic formula in [9]. The tune, $\nu$, is 61.4 .

The situation for injection and extraction is slightly different. We start with damped bunches assumed to have zero displacement, and inject bunches with random offsets. The injected bunches generate transverse wake forces that start to perturb the damped bunches; initially, at least, the perturbations are random. The subsequent development may then follow that described above, with small number of modes (those with the largest growth rates) becoming dominant.

In order to confirm this explanation for the modulation observed in Fig. 2, we need to be able to compute the frequencies of the peaks shown in Fig. 3. If our conjecture is correct, these peaks should correspond to the modes with the largest growth rates. Consider a mode number, $\mu$. The displacement of bunch $m$ is given by [17]

$$
y_{m}(t)=\exp \left[i\left(\frac{2 \pi m \mu}{M}-\omega_{\beta} t\right)\right] .
$$

This has the form of a traveling wave, sampled at discrete locations indexed by the bunch number, and in a frame of reference that is the rest frame of the bunches.

Consider a fixed location in the ring (in the laboratory frame). Assume that the bunches are moving to the right. Consider the special case when the mode number $\mu$ is equal and opposite to the tune, i.e. $\mu=-\nu$. Then, since the betatron frequency is related to the tune by

$$
\omega_{\beta}=2 \pi \nu / T_{0},
$$

we see that the wave is moving to the left (in the beam frame) at the same speed as the bunches are moving to the right (in the laboratory frame). In the laboratory frame, the wave appears to be frozen.

Now consider the dominant mode. The number of this mode, say $\mu_{D}$, is the largest integer smaller than $-\nu$ [17]. This is illustrated in Fig. 8, where $\mu_{D}$ is -62 . The differ- ence, which is just the fractional part of the tune, is a small number, so a wave corresponding to this mode number moves slowly, relative to the rate of revolution. The difference between the dominant mode number and the tune then gives the number of oscillations observed at the extraction location over the period of one turn. The vertical tune for the lattice used in Sec. III is 61.4. For this tune, the dominant mode number is -62 . Comparing with -61.4 gives a frequency of 0.6 cycles per turn. This is exactly equal to the frequency of the highest peak in Fig. 3. The frequency of the next peak is obtained by taking the difference between the next dominant mode number, -61 , and the tune. This gives 1.6, which is the correct frequency for the next peak in Fig. 3. In this way, we see that the frequencies for the other peaks can be obtained, since they are all at intervals of 1 cycle per turn.

We now follow this with a more rigorous analysis. Define $M$ fixed locations around the ring. At time $t$, the bunch number seen at the extraction location, say $n$, is

$$
m=n-t / \tau \text {. }
$$

Note that there is a bunch at the location only when $t$ is a multiple of $\tau$. Otherwise, there would just be the empty space between bunches. Note also that we only need to consider $\mu$ from 0 to $M-1$. An integer of $\mu$ outside this range would give the same mode as an integer inside the range that differs by a multiple of $M$, because of the periodic nature of Eq. (B1). Substituting Eq. (B3) into Eq. (B1), the displacement of a bunch at the extraction location is then given by

$$
y(t)=\exp \left[i\left(\frac{2 \pi(n-t / \tau) \mu}{M}-\omega_{\beta} t\right)\right] .
$$

The time to travel one bunch spacing is related to the time for one revolution by

$$
\tau=\frac{T_{0}}{M}
$$

Substituting and rearranging gives

$$
y(t)=\exp \left[i\left(\frac{2 \pi n \mu}{M}-\frac{2 \pi(\mu+\nu) t}{T_{0}}\right)\right] .
$$

Let the fractional part of the tune be $\Delta$. Let the dominant mode number be $\mu_{D}$. These are related to the tune by

$$
\mu_{D}=M-(n-\Delta+1) .
$$

Note that we need to take the upper fractional part for [17], i.e. the difference between the tune and the smallest integer greater than the tune. This is because of the nature of the expression for the growth rate in the usual macroparticle model [17]. Assuming that the dominant mode is much larger than other modes,

$$
y(t) \approx \exp \left(i\left[\frac{2 \pi n \mu_{D}}{M}-\frac{2 \pi\left(\mu_{D}+\nu\right) t}{T_{0}}\right]\right) .
$$


Substituting Eq. (B7) into Eq. (B8) gives

$$
y(t) \approx \exp \left(i\left[\frac{2 \pi n \mu_{D}}{M}-\frac{2 \pi(M+\Delta-1) t}{T_{0}}\right]\right) .
$$

The bunch displacement at the extraction location is observed only when a bunch arrives there. So the only times that are meaningful are when

$$
t=p \tau
$$

where $p$ is an integer. Substituting into Eq. (B9) gives

$$
y(t) \approx \exp \left[i\left(\frac{2 \pi n \mu_{D}}{M}-\frac{2 \pi M p \tau}{T_{0}}-\frac{2 \pi(\Delta-1) t}{T_{0}}\right)\right] .
$$

Since $T_{0}=M \tau$,

$$
y(t) \approx \exp \left[i\left(\frac{2 \pi n \mu_{D}}{M}-2 \pi M p-\frac{2 \pi(\Delta-1) t}{T_{0}}\right)\right] .
$$

The only time dependent factor here is

$$
y(t) \approx \exp \left[i \frac{2 \pi(1-\Delta) t}{T_{0}}\right] .
$$

This has a frequency of $(1-\Delta)$ cycles per turn. For the tune of 61.4 , this is 0.6 cycles per turn, exactly what is observed in the jitter spectrum in Fig. 3.

Other growth modes have mode numbers, $\mu$, that are smaller than $\mu_{D}$. Repeating the above steps with $\mu$ gives

$$
y(t) \approx \exp \left[i \frac{2 \pi\left(\mu_{D}-\mu+1-\Delta\right) t}{T_{0}}\right] .
$$

This has a frequency of $\left(\mu_{D}-\mu+1-\Delta\right)$ cycles per turn. The dominant mode number $\mu_{D}$ is -62 . Thus, the mode number of -61 would give a frequency of 1.6, the mode number of -60 would give a frequency of 2.6 , and so on.

The correct frequencies are thus obtained. This strongly suggests that the Fourier modes can indeed give rise to modulation in the extraction jitter. It is also interesting to note that, although comparisons of different cases in Fig. 8 show that the real lattice has a significant impact on the growth rates, the trends in growth rates appear similar to the uniform focusing case. We have predicted in [13] that the growth modes can mix into the decay modes and cause the decay modes to grow. The results in Fig. 3 support this conclusion. For uniform focusing strength, there should be no peak with negative frequencies, since these would correspond to decay modes with mode numbers $-63,-64$, and so on as shown in Fig. 8. The presence of peaks with these mode numbers in Fig. 3 shows that the decay modes have indeed grown.

[1] K. Kubo et al., Phys. Rev. Lett. 88, 194801 (2002).

[2] Y. Honda et al., Phys. Rev. Lett. 92, 054802 (2004).

[3] A. Andersson et al., Nucl. Instrum. Methods Phys. Res., Sect. A 591, 437 (2008).

[4] J. Byrd et al., Phys. Rev. Lett. 79, 79 (1997).

[5] M. Izawa, Y. Sato, and T. Toyomasu, Phys. Rev. Lett. 74, 5044 (1995).

[6] S. Prabhakar, J. D. Fox, and D. Teytelman, Phys. Rev. Lett. 86, 2022 (2001).

[7] R. L. Gluckstern, H. Okamoto, and B. Zotter, Phys. Rev. E 52, 1026 (1995).

[8] ILC Reference Design Report, http://www.linearcollider. org/cms/?pid=1000437, 2007.

[9] https://wiki.lepp.cornell.edu/ilc/bin/view/Public/ DampingRings/WebHome.

[10] SuperB Conceptual Design Report No. SLAC-R-856, http://www.pi.infn.it/SuperB/?q=CDR.

[11] H. Ohkuma, Proceedings of the 11th European Particle Accelerator Conference, Genoa, 2008 (EPS-AG, Genoa, Italy, 2008), pp. 36-40.

[12] L. Vos, CERN Report No. CERN-AB-2003-093, 2003; B. Zotter, CERN Report No. CERN-AB-2005-043, 2005; A. M. Al-Khateeb et al., Phys. Rev. ST Accel. Beams 10, 064401 (2007).

[13] K. M. Hock and A. Wolski, Phys. Rev. ST Accel. Beams 10, 084401 (2007).

[14] A. Koschik, in Proceedings of the 9th European Particle Accelerator Conference, Lucerne, 2004 (EPS-AG, Lucerne, 2004), p. 2490; BNL Accelerator Physics Seminar http://www.agsrhichome.bnl.gov/AP/ APseminar.html, 2004.

[15] J. A. Clarke et al., in Proceedings of the 11th European Particle Accelerator Conference, Genoa, 2008 (Ref. [11]), pp. 1915-1917.

[16] M. Kuriki et al., ILC-Asia Notes Report No. 2006-03, 2006, http://lcdev.kek.jp/ILCAsiaNotes/.

[17] A. W. Chao, Physics of Collective Beam Instabilities (John Wiley \& Sons, New York, 1993), pp. 38-117 and 203211.

[18] M. Venturini et al., in Proceedings of the 2007 Particle Accelerator Conference, Albuquerque, New Mexico, 2007 (IEEE, Albuquerque, New Mexico, 2007), pp. 4141-4143.

[19] N. Nakamura and T. Koseki, Proceedings of the Particle Accelerator Conference, Vancouver, BC, Canada, 1997 (IEEE, New York, 1997), pp. 1756-1758.

[20] S.S. Win et al., in Proceedings of the Asian Particle Accelerator Conference, Beijing, 2001 (Institute of High Energy, Physics, Beijing, 2001), pp. 380-382.

[21] K. A. Thompson and R. D. Ruth, Phys. Rev. D 43, 3049 (1991).

[22] W. H. Press et al., Numerical Recipes in C (Cambridge University Press, Cambridge, England, 1997), pp. 538545 , 2nd ed. 